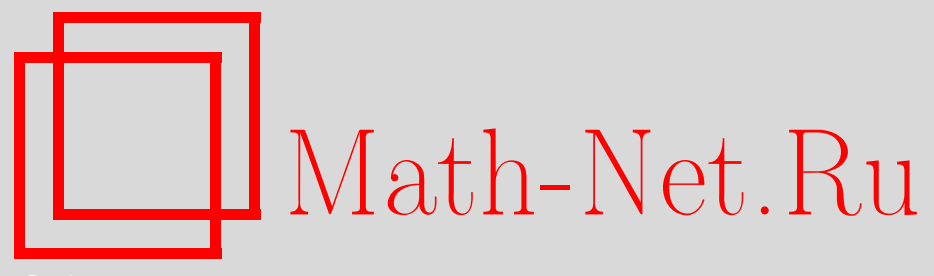

В. А. Юрко, Об обратной задаче для квазипериодических дифференциальных пучков с условиями разрыва внутри интервала, Матем. заметки, 2015, том 98, выпуск $3,476-480$

DOI: https://doi.org/10.4213/mzm10820

Использование Общероссийского математического портала Math-Net.Ru подразумевает, что вы прочитали и согласны с пользовательским соглашением http://www . mathnet.ru/rus/agreement

Параметры загрузки:

IP : 54.166 .219 .16

26 апреля 2023 г., 15:12:00

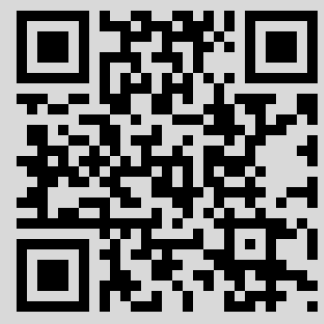




\section{Об обратной задаче для квазипериодических дифференциальных пучков с условиями разрыва внутри интервала}

\section{В. А. Юрко}

1. Рассмотрим краевую задачу $B$ вида

$$
\begin{gathered}
y^{\prime \prime}+\left(\rho^{2}+\rho p(x)+q(x)\right) y=0, \quad x \in[0, T], \\
y(0)=\alpha y(T), \quad y^{\prime}(0)-\left(i \rho h^{\prime}+h\right) y(0)=\beta y^{\prime}(T), \\
y\left(b_{j}+0\right)=\gamma_{j} y\left(b_{j}-0\right), \\
y^{\prime}\left(b_{j}+0\right)=\gamma_{j}^{-1} y^{\prime}\left(b_{j}-0\right)+\left(i \rho \eta_{j}^{\prime}+\eta_{j}\right) y\left(b_{j}-0\right), \quad j=1, \ldots, N-1, \\
0=b_{0}<b_{1}<\cdots<b_{N-1}<b_{N}=T,
\end{gathered}
$$

где $\rho$ - спектральный параметр, $p(x), q(x)$ - комплекснозначные функции, $h^{\prime}, h, \alpha, \beta, \gamma_{j}$, $\eta_{j}^{\prime}, \eta_{j}-$ комплексные числа, причем

$$
\alpha \beta \gamma_{j} \neq 0, \quad z_{0}^{ \pm}:=\alpha\left(1 \mp h^{\prime}\right)+\beta \neq 0, \quad \xi_{j}^{ \pm}:=\frac{\gamma_{j}+\left(\gamma_{j}\right)^{-1}}{2} \mp \frac{\eta_{j}^{\prime}}{2} \neq 0 .
$$

Предположим, что $p(x) \in A C[0, T]$ и $q(x) \in L(0, T)$. Исследуется нелинейная обратная задача восстановления $B$ по спектральным данным. Для этой обратной задачи доказана теорема единственности и получена процедура построения решения.

Краевые задачи с условиями разрыва внутри интервала часто возникают в математике, механике, физике, геофизике и других областях естествознания. Как правило, такие задачи связаны с разрывными свойствами среды (см., например, [1]-[3] и литературу там). Обратные спектральные задачи этого типа играют также важную роль при исследовании некоторых нелинейных эволюционных уравнений математической физики. Важным стимулом для изучения краевой задачи $B$ является тот факт, что обратная задача для $B$, рассматриваемая в этой статье, возникает в теории обратных задач для дифференциальных операторов на пространственных сетях (графах) с циклами, которые имеют много приложений в естествознании и технике. Точнее, обратные спектральные задачи на графах с циклами сводятся к решению обратной задачи для $B$, рассматриваемой в данной статье.

2. Пусть $S(x, \rho)$ и $C(x, \rho)$ - решения уравнения (1), удовлетворяющие условиям (3) и начальным условиям

$$
S(0, \rho)=C^{\prime}(0, \rho)=0, \quad S^{\prime}(0, \rho)=C(0, \rho)=1 .
$$

Положим

$$
\varphi(x, \rho)=C(x, \rho)+\left(i \rho h^{\prime}+h\right) S(x, \rho), \quad d(\rho)=S(T, \rho), \quad d_{1}(\rho)=C(T, \rho) .
$$

Собственные значения $\mathcal{P}=\left\{\rho_{n}\right\}_{n \in \mathbb{Z}}$ краевой задачи (1)-(3) совпадают с нулями характеристической функции

$$
a(\rho)=\alpha \varphi(T, \rho)+\beta S^{\prime}(T, \rho)-(1+\alpha \beta) .
$$

Пусть $\Lambda:=\{n: n= \pm 1, \pm 2, \ldots\}=\mathbb{Z} \backslash\{0\}$, и пусть $\mathcal{V}=\left\{\nu_{n}\right\}_{n \in \Lambda}-$ нули функции $d(\rho)$. Тогда $\left\{\nu_{n}\right\}_{n \in \Lambda}$ являются собственными значениями краевой задачи $\mathcal{B}$ для уравнения (1)

Работа выполнена при поддержке Минобрнауки РФ (проект 1.1436.2014K) и Российского фонда фундаментальных исследований (грант 13-01-00134).

DOI: $10.4213 / \mathrm{mzm} 10820$ 
с условиями (3) и с краевыми условиями $y(0)=y(T)=0$. Без ограничения общности считаем, что нумерация выбрана так, что $\nu_{n} \neq \nu_{k}$, если $n k<0$. Пусть $m_{n}-$ кратность $\nu_{n}$ $\left(\nu_{n}=\nu_{n+1}=\cdots=\nu_{n+m_{n}-1}\right)$. Положим

$$
I:=\left\{n \in \Lambda: \nu_{n-1} \neq \nu_{n}\right\}, \quad I^{\prime}:=\left\{n \in I: m_{n}>1\right\} .
$$

Обозначим

$$
D(\rho)=\alpha \varphi(T, \rho)+\beta S^{\prime}(T, \rho), \quad Q(\rho)=\alpha \varphi(T, \rho)-\beta S^{\prime}(T, \rho) .
$$

Тогда

$$
D(\rho)=d(\rho)+(1+\alpha \beta) .
$$

Так как $\varphi(x, \rho) S^{\prime}(x, \rho)-\varphi^{\prime}(x, \rho) S(x, \rho) \equiv 1$, то

$$
Q^{2}(\rho)=D^{2}(\rho)-4 \alpha \beta\left(1+\varphi^{\prime}(T, \rho) S(T, \rho)\right)
$$

и, следовательно,

$$
\dot{Q}(\rho) Q(\rho)=\dot{D}(\rho) D(\rho)-2 \alpha \beta\left(\dot{\varphi}^{\prime}(T, \rho) S(T, \rho)+\varphi^{\prime}(T, \rho) \dot{S}(T, \rho)\right),
$$

где "точка" обозначает дифференцирование по $\rho$. Пусть $n \in I$. Положим

$$
\omega_{n}= \begin{cases}0, & Q\left(\nu_{n}\right)=0, \\ +1, & Q\left(\nu_{n}\right) \neq 0, \arg Q\left(\nu_{n}\right) \in[0, \pi), \\ -1, & Q\left(\nu_{n}\right) \neq 0, \arg Q\left(\nu_{n}\right) \in[\pi, 2 \pi),\end{cases}
$$

где

$$
\omega_{n \nu}:=d_{1}^{(\nu)}\left(\nu_{n}\right), \quad \nu=0, \ldots, m_{n}-1, \quad I_{0}=\left\{n \in I^{\prime}: \omega_{n}=0\right\}, \quad I_{1}=\left\{n \in I^{\prime}: \omega_{n} \neq 0\right\} .
$$

Последовательность $\Omega=\left\{\omega_{n}\right\}_{n \in I} \cup\left\{\omega_{n \nu}\right\}_{n \in I_{0}, \nu=1, \ldots, m_{n}-1}$ называется $\Omega$-последовательностъю для $B$. Отметим, что если $I^{\prime}=\varnothing$ (т.е. $m_{n}=1$ при всех $\left.n\right)$, то $\Omega=\left\{\omega_{n}\right\}_{n \in \Lambda}$.

Пусть $\alpha, \beta$ и $\gamma_{j}$ известны априори и фиксированы. Обратная задача ставится следующим образом.

ОБРАТНАЯ ЗАДАЧА 1 . Даны $a(\rho), d(\rho) u \Omega$; nостроить $B$.

Отметим, что, вообще говоря, невозможно восстановить все коэффициенты из (2), (3). Эта обратная задача является обобщением классических обратных задач для операторов Штурма-Лиувилля [4].

3. Пусть $\Phi(x, \rho)$ - решение уравнения (1) с условиями (3) и краевыми условиями

$$
\Phi(0, \rho)=1, \quad \Phi(T, \rho)=0 .
$$

Обозначим $M(\rho):=\Phi^{\prime}(0, \rho)$. Функция $M(\rho)$ называется функцией Вейля. Ясно, что

$$
\begin{gathered}
\Phi(x, \rho)=C(x, \rho)+M(\rho) S(x, \rho), \\
M(\rho)=-\frac{d_{1}(\rho)}{d(\rho)} .
\end{gathered}
$$

Обозначим $T_{k}:=b_{k}-b_{k-1}, k=1, \ldots, N$. Тогда

$$
b_{k}=T_{1}+\cdots+T_{k}, \quad T=T_{1}+\cdots+T_{N} .
$$

Положим $x_{k}=x-b_{k-1}$ при $x \in\left[b_{k-1}, b_{k}\right]$; следовательно, $x_{k} \in\left[0, T_{k}\right]$. Пусть $S_{k}\left(x_{k}, \rho\right)$ и $C_{k}\left(x_{k}, \rho\right)$ - решения уравнения $(1)$ на $\left[b_{k-1}, b_{k}\right]$ при начальных условиях

$$
S_{k}(0, \rho)=C_{k}^{\prime}(0, \rho)=0, \quad S_{k}^{\prime}(0, \rho)=C_{k}(0, \rho)=1 .
$$


Лемма 1. При $k=1, \ldots, N-1, \nu=0,1$ справедливы соотношения

$$
\begin{array}{rl}
S^{(\nu)}\left(b_{k+1}-0, \rho\right)=\gamma_{k} & S\left(b_{k}-0, \rho\right) C_{k+1}^{(\nu)}\left(T_{k+1}, \rho\right)+\gamma_{k}^{-1} S^{\prime}\left(b_{k}-0, \rho\right) S_{k+1}^{(\nu)}\left(T_{k+1}, \rho\right) \\
+ & \left(i \rho \eta_{k}^{\prime}+\eta_{k}\right) S\left(b_{k}-0, \rho\right) S_{k+1}^{(\nu)}\left(T_{k+1}, \rho\right) \\
C^{(\nu)}\left(b_{k+1}-0, \rho\right)=\gamma_{k} C\left(b_{k}-0, \rho\right) C_{k+1}^{(\nu)}\left(T_{k+1}, \rho\right)+\gamma_{k}^{-1} C^{\prime}\left(b_{k}-0, \rho\right) S_{k+1}^{(\nu)}\left(T_{k+1}, \rho\right) \\
+\left(i \rho \eta_{k}^{\prime}+\eta_{k}\right) C\left(b_{k}-0, \rho\right) S_{k+1}^{(\nu)}\left(T_{k+1}, \rho\right) .
\end{array}
$$

В самом деле, зафиксируем $k=1, \ldots, N-1$. Пусть $x \in\left[b_{k}, b_{k+1}\right]$, т.е.

$$
x=x_{k+1}+b_{k}, \quad x_{k+1} \in\left[0, T_{k+1}\right] .
$$

Используя фундаментальную систему решений $C_{k+1}\left(x_{k+1}, \rho\right), S_{k+1}\left(x_{k+1}, \rho\right)$, получаем

$$
S^{(\nu)}(x, \rho)=A(\rho) C_{k+1}^{(\nu)}\left(x_{k+1}, \rho\right)+B(\rho) S_{k+1}^{(\nu)}\left(x_{k+1}, \rho\right), \quad \nu=0,1 .
$$

Учитывая начальные условия (10), находим коэффициенты $A(\rho)$ и $B(\rho)$, и приходим к (11). Соотношение (12) доказывается аналогично.

Обозначим

$$
\begin{aligned}
\mathcal{E}(x) & =\frac{1}{2} \int_{0}^{x} p(t) d t, \quad \omega=\frac{1}{2 T} \int_{0}^{T} p(t) d t, \\
\Pi^{ \pm}=\{\rho: \pm \tau>0\}, & \Pi_{\delta}^{+}=\{\rho: \arg \rho \in[\delta, \pi-\delta]\}, \quad \Pi_{\delta}^{-}=\{\rho: \arg \rho \in[\pi+\delta, 2 \pi-\delta]\} .
\end{aligned}
$$

Известно (см. [4]), что существует фундаментальная система решений $\left\{Y_{1}^{ \pm}(x, \rho), Y_{2}^{ \pm}(x, \rho)\right\}$, $x \in[0, T], \rho \in \Pi^{ \pm}$, уравнения (1) такая, что функции $Y_{k}^{ \pm}(x, \rho)$ регулярны при $\rho \in \Pi^{ \pm},|\rho|>\rho^{*}$, непрерывны при $x \in[0, T], \rho \in \overline{\Pi^{ \pm}},|\rho| \geqslant \rho^{*}$, и при $|\rho| \rightarrow \infty, \rho \in \overline{\Pi^{ \pm}}, k=1,2, \nu=0,1$,

$$
\left.\frac{d^{\nu}}{d x^{\nu}} Y_{k}^{ \pm}(x, \rho)=\left(\rho R_{k}\right)^{\nu} \exp (\rho x+\mathcal{E}(x)) R_{k}\right)[1], \quad[1]=1+O\left(\rho^{-1}\right), \quad R_{k}=(-1)^{k-1} i .
$$

Используя (13) и лемму 1 , получаем при $x \in\left(b_{j}, b_{j+1}\right),|\rho| \rightarrow \infty, \rho \in \Pi_{\delta}^{ \pm}$

$$
\begin{aligned}
C^{(\nu)}(x, \rho) & =\frac{\xi_{1}^{ \pm} \ldots \xi_{j}^{ \pm}}{2}(\mp i \rho)^{\nu} \exp (\mp i(\rho x+\mathcal{E}(x))[1], \\
S^{(\nu)}(x, \rho) & =\mp \frac{\xi_{1}^{ \pm} \ldots \xi_{j}^{ \pm}}{2 i \rho}(\mp i \rho)^{\nu} \exp (\mp i(\rho x+\mathcal{E}(x))[1], \\
\Phi^{(\nu)}(x, \rho) & =\frac{1}{\xi_{1}^{ \pm} \ldots \xi_{j}^{ \pm}}( \pm i \rho)^{\nu} \exp ( \pm i(\rho x+\mathcal{E}(x))[1],
\end{aligned}
$$

Из (14)-(16) выводим, что при $|\rho| \rightarrow \infty, \rho \in \Pi_{\delta}^{ \pm}$

$$
\begin{aligned}
& a(\rho)=\frac{z_{0}^{ \pm}}{2}\left(\xi_{1}^{ \pm} \ldots \xi_{N-1}^{ \pm}\right) \exp (\mp i(\rho+\omega) T)[1], \\
& d(\rho)=\mp \frac{1}{2 i \rho}\left(\xi_{1}^{ \pm} \ldots \xi_{N-1}^{ \pm}\right) \exp (\mp i(\rho+\omega) T)[1] .
\end{aligned}
$$

Пусть $n \in I$. Используя (9), получаем, что в окрестности точки $\rho=\nu_{n}$ функция $M(\rho)$ имеет представление

$$
M(\rho)=\sum_{\nu=0}^{m_{n}-1} \frac{M_{n+\nu}}{\left(\rho-\nu_{n}\right)^{\nu+1}}+M_{n}^{*}(\rho),
$$


где $M_{n}^{*}(\rho)$ регулярна при $\rho=\nu_{n}$, а коэффициенты $M_{n+\nu}, \nu=0, \ldots, m_{n}-1$ вычисляются по $d_{1}^{(\nu)}\left(\nu_{n}\right)$ и $d^{\left(\nu+m_{n}\right)}\left(\nu_{n}\right)$ при $\nu=0, \ldots, m_{n}-1$. Точнее,

$$
\begin{gathered}
M_{n+m_{n}-1-\nu}=-\frac{1}{d_{0 n}}\left(d_{\nu n}^{1}+\sum_{k=0}^{\nu-1} M_{n+m_{n}-1-k} d_{\nu-k, n}\right), \quad n \in I, \quad \nu=0, \ldots, m_{n}-1, \\
d_{\nu n}^{1}:=\frac{1}{\nu !} d_{1}^{(\nu)}\left(\nu_{n}\right), \quad d_{\nu n}:=\frac{1}{\left(\nu+m_{n}\right) !} d^{\left(\nu+m_{n}\right)}\left(\nu_{n}\right), \quad \nu=0, \ldots, m_{n}-1 .
\end{gathered}
$$

В частности, $M_{n+m_{n}-1}=-d_{0 n}^{1} / d_{0 n}$. Если $m_{n}=1$ (т.е. $n \in I \backslash I^{\prime}$ ), то

$$
M_{n}=-\frac{d_{1}\left(\nu_{n}\right)}{\dot{d}\left(\nu_{n}\right)} .
$$

Последовательность $\left\{M_{n}\right\}_{n \in \Lambda}$ называется последовательностъю Вейля. Совокупность $\mathcal{D}=$ $\left\{\nu_{n}, M_{n}\right\}_{n \in \Lambda}$ называется спектральными данными.

Рассмотрим следующую вспомогательную обратную задачу 2.

ОБРАТНАЯ ЗАДАЧА 2. Заданы спектралъные данные

$$
\mathcal{D}=\left\{\nu_{n}, M_{n}\right\}_{n \in \Lambda}, \quad \gamma_{j}, \quad j=1, \ldots, N-1 ;
$$

nocmpoums $p(x), q(x), x \in(0, T), \eta_{j}^{\prime}, \eta_{j}, j=1, \ldots, N-1$.

Эта обратная задача решена в [5], где доказана теорема единственности и указана процедура построения решения.

4. Пусть даны $a(\rho), d(\rho)$ и $\Omega$. Напомним, что $\alpha, \beta, \gamma_{j}, j=1, \ldots, N-1$ известны априори. Решение обратной задачи 1 строится следующим образом.

Сначала находим нули $\mathcal{V}=\left\{\nu_{n}\right\}_{n \in \Lambda}$ функции $d(\rho)$. Используя асимптотику (17), вычисляем $\left(\xi_{1}^{ \pm} \ldots \xi_{N-1}^{ \pm}\right)$и $h^{\prime}$. Учитывая $(5)$, строим $D(\rho)$. В силу (6) имеем $Q^{2}\left(\nu_{n}\right)=D^{2}\left(\nu_{n}\right)-4 \alpha \beta$ и

$$
Q\left(\nu_{n}\right)=\omega_{n} \sqrt{D^{2}\left(\nu_{n}\right)-4 \alpha \beta}, \quad n \in I .
$$

Здесь и далее, если $z=|z| e^{i \xi}, \xi \in[0,2 \pi)$, то $\sqrt{z}=|z|^{1 / 2} e^{i \xi / 2}$. Так как $\omega_{n 0}=d_{1}\left(\nu_{n}\right)=\varphi\left(T, \nu_{n}\right)$, вычисляем $\omega_{n 0}$ по формуле

$$
\omega_{n 0}=\frac{D\left(\nu_{n}\right)+Q\left(\nu_{n}\right)}{2 \alpha} .
$$

Построим последовательность Вейля $\left\{M_{n}\right\}_{n \in \Lambda}$ следующим образом.

Случай 1 . Пусть $n \in I \backslash I^{\prime}$ (т.е. $m_{n}=1$ ). Тогда в силу (19) имеем

$$
M_{n}=-\frac{\omega_{n 0}}{\dot{d}\left(\nu_{n}\right)} .
$$

Случай 2. Пусть $n \in I_{1}$ (т.е. $m_{n}>1, \omega_{n} \neq 0$ ). Тогда из (7) вытекает

$$
(\dot{Q}(\rho) Q(\rho))_{\mid \rho=\nu_{n}}^{(\nu-1)}=(\dot{D}(\rho) D(\rho))_{\mid \rho=\nu_{n}}^{(\nu-1)}, \quad \nu=1, \ldots, m_{n}-1 .
$$

Используя (23), находим $Q^{(\nu)}\left(\nu_{n}\right), \nu=1, \ldots, m_{n}-1$. Так как

$$
d_{1}(\rho)=\frac{D(\rho)+Q(\rho)}{2 \alpha}-\left(i \rho h^{\prime}+h\right) d(\rho),
$$

вычисляем $\omega_{n \nu}, \nu=1, \ldots, m_{n}-1$, по формуле

$$
\omega_{n \nu}=\frac{D^{(\nu)}\left(\nu_{n}\right)+Q^{(\nu)}\left(\nu_{n}\right)}{2 \alpha}, \quad \nu=1, \ldots, m_{n}-1 .
$$

Случай 3. Пусть $n \in I_{0}$ (т.е. $m_{n}>1, \omega_{n}=0$ ). Тогда $\omega_{n \nu}, \nu=1, \ldots, m_{n}-1$, даны априори.

Таким образом, мы построили последовательность Вейля $\left\{M_{n}\right\}_{n \in \Lambda}$. Решая вспомогательную обратную задачу 2 , находим $p(x), q(x), x \in(0, T), \eta_{j}^{\prime}, \eta_{j}, j=1, \ldots, N-1$, а затем вычисляем $h$, используя (4). Таким образом, справедлива следующая теорема. 
Теорема 1. Задание $a(\rho), d(\rho)$ и $\Omega$ однозначно определяет краевую задачу В. Решение обратной задачи 1 может быть найдено по следующему алгоритму.

Алгоритм 1. Даны $а(\rho), d(\rho) u \Omega$.

1) Вычисляем нули $\mathcal{V}=\left\{\nu_{n}\right\}_{n \in \Lambda}$ функиии $d(\rho)$.

2) Находим $h^{\prime}$, используя (17).

3) Строим $D(\rho)$ по формуле (5).

4) Вычисляем $Q^{2}\left(\nu_{n}\right)=D^{2}\left(\nu_{n}\right)-4 \alpha \beta, u Q\left(\nu_{n}\right)$ nо (20).

5) Строим $\omega_{n 0}$ согласно (21).

6) Находим $Q^{(\nu)}\left(\nu_{n}\right), n \in I_{1}, \nu=1, \ldots, m_{n}-1$, используя (23).

7) Строим $\omega_{n \nu}, n \in I_{1}, \nu=1, \ldots, m_{n}-1$, по формуле (24).

8) Вычисляем последовательность Вейля $\left\{M_{n}\right\}_{n \geqslant 1}$, используя (18) и (22).

9) Находим $p(x), q(x), x \in(0, T) u \eta_{j}^{\prime}, \eta_{j}, j=1, \ldots, N-1$, решая вспомогательную обратную задачу 2.

10) Вычисляем коэффиииент $h$, используя (4).

\section{СПИСОК ЦИТИРОВАННОЙ ЛИТЕРАТУРЫ}

[1] F. Lapwood, T. Usami, Free Oscillations of the Earth, Cambridge Univ. Press, Cambridge, 1981. [2] R. J. Krueger, J. Math. Phys., 23:3 (1982), 396-404. [3] V. A. Yurko, Integral Transform. Spec. Funct., 10:2 (2000), 141-164. [4] G. Freiling, V. Yurko, Inverse Sturm-Liouville Problems and their Applications, NOVA Sci. Publ., New York, 2001. [5] V. A. Yurko, Anal. Math. Phys., 2:3 (2012), 215-230.

\section{В. А. Юрко}

Поступило

Саратовский государственный

университет имени Н.Г. Чернышевского

E-mail: yurkova@info.sgu.ru 